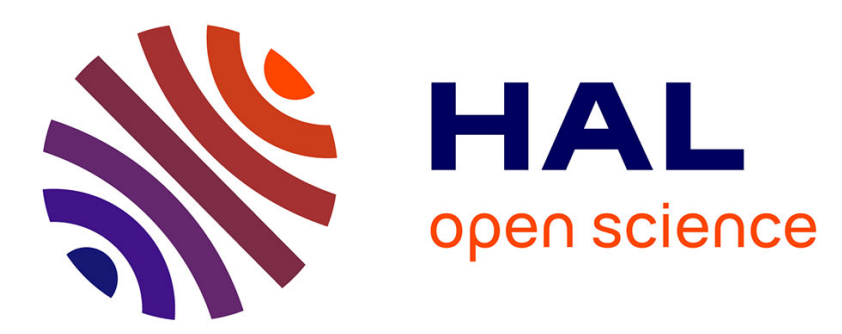

\title{
A Criterion for Crack Kinking Out of an Interface
}

Dominique Leguillon, Sébastien Murer

\section{To cite this version:}

Dominique Leguillon, Sébastien Murer. A Criterion for Crack Kinking Out of an Interface. Key Engineering Materials, 2008, 385-387, pp.9-12. 10.4028/www.scientific.net/KEM.385-387.9 . hal01509353

\section{HAL Id: hal-01509353 \\ https://hal.science/hal-01509353}

Submitted on 17 Apr 2017

HAL is a multi-disciplinary open access archive for the deposit and dissemination of scientific research documents, whether they are published or not. The documents may come from teaching and research institutions in France or abroad, or from public or private research centers
L'archive ouverte pluridisciplinaire HAL, est destinée au dépôt et à la diffusion de documents scientifiques de niveau recherche, publiés ou non, émanant des établissements d'enseignement et de recherche français ou étrangers, des laboratoires publics ou privés.

\section{(c)(1)}

Distributed under a Creative Commons Attribution| 4.0 International License 


\title{
A criterion for crack kinking out of an interface
}

\author{
Dominique Leguillon ${ }^{a}$, Sébastien Murer ${ }^{b}$ \\ ${ }^{a, b}$ Institut Jean Le Rond d'Alembert - CNRS UMR7190 \\ Université Pierre et Marie Curie \\ 4 place Jussieu, 75005 PARIS - FRANCE \\ aleguillo@Imm.jussieu.fr , ${ }^{\mathrm{b}}$ murer@Imm.jussieu.fr
}

Keywords: Brittle fracture, interfaces, crack kinking, matched asymptotics.

\begin{abstract}
Cotterell and Rice theory (1980) on the kinking of a crack submitted to a biaxial loading in a homogeneous material has been recently revisited (Leguillon and Murer 2008). The mixed criterion for fracture which involves both an energetic and a stress condition (Leguillon 2002) allows defining a positive threshold of the $T$-stress below which no branching can occur (Selvarathinam and Goree 1998). This analysis enters within a more general mixed-mode analysis $(I+I I+T$-stress). Despite the complex terms and the oscillations, results extend to interfacial cracks. The assumption of a crack jump as a consequence of the energy balance allows getting rid of the problem brought by the oscillations due to these complex terms. This approach brings a new insight on the prediction of crack kinking out of a bimaterial interface.
\end{abstract}

\section{Introduction}

Within the plane strain elasticity framework, the displacement field in the vicinity of a crack tip at the interface between two homogeneous materials can be described by the so-called Williams' series made of power terms (1). The leading terms involve a complex singularity exponent $\lambda=1 / 2+\mathrm{i} \varepsilon$ associated with a complex mode $\underline{u}$ and the conjugates. The first non singular term called " $T$-stress" corresponds to a tension acting in a direction parallel to the crack. The related power is 1 ; the associated mode is denoted $\underline{t}$ and $T$ holds for the corresponding intensity factor, i.e. the remote tension

$$
\left\{\begin{array}{l}
\underline{U}^{0}(r, \theta)=\underline{R}+K r^{1 / 2+\mathrm{i} \varepsilon} \underline{u}(\theta)+\bar{K} r^{1 / 2-\mathrm{i} \varepsilon} \underline{\bar{u}}(\theta)+T r \underline{t}(\theta)+\ldots \\
\underline{\underline{\sigma}}^{0}(r, \theta)=K r^{-1 / 2+\mathrm{i} \varepsilon} \underline{\underline{s}}(\theta)+\bar{K} r^{-1 / 2-\mathrm{i} \varepsilon} \underline{\underline{s}}(\theta)+T \underline{\underline{\tau}}(\theta)+\ldots
\end{array}\right.
$$

where $r$ and $\theta$ are the polar coordinates, with the crack tip $O$ chosen as origin. The meaning of index 0 will be explained in the next section. The constant vector $\underline{R}$ is for consistency only. The dots refer to modes with higher order real parts $(3 / 2,2,5 / 2 \ldots)$. Oscillations are due to the terms $r^{ \pm \mathrm{i} \varepsilon}$ and $(1)_{2}$ bring into evidence the singular behaviour in the vicinity of the crack tip.

It is worth noting that many authors ([3], [5], [6]) emphasize on the difficulty to extract $T$ from a finite element approximation of (1), even in the real homogeneous case. They propose different procedures leading to quite large inaccuracies. Herein, the path-independent integral $H$ ([4], [9]) is used and proved to be a simple and accurate procedure to determine either the real intensity factor $T$ or the complex one $K$.

Introducing dimensionless mix mode parameters

$$
m(r)=\frac{\bar{K}}{K} r^{-2 \mathrm{i} \varepsilon} ; M(r)=\frac{T}{K} r^{1 / 2-\mathrm{i} \varepsilon}
$$

equation (1) can be rewritten as follows

$$
\left\{\begin{array}{l}
\underline{U}^{0}(r, \theta)=\underline{R}+K r^{1 / 2+\mathrm{i} \varepsilon}(\underline{u}(\theta)+m(r) \underline{\bar{u}}(\theta)+M(r) \underline{t}(\theta)+\ldots) \\
\underline{\underline{\sigma}}^{0}(r, \theta)=K r^{-1 / 2+\mathrm{i} \varepsilon}(\underline{\underline{s}}(\theta)+m(r) \underline{\underline{s}}(\theta)+M(r) \underline{\underline{\tau}}(\theta)+\ldots)
\end{array}\right.
$$


See [2] and [10] for the $r$ dependency of $m$. The parameter $M$ depends on $r$ as well and is a generalisation of the so-called "stress biaxiality ratio" $B$ used by Leevers and Radon [6].

The aim of this paper is to extend Leguillon's criterion ([7], [12]) in order to include the $T$-stress in the analysis. This criterion is a combination of energy and stress conditions which avoids any arbitrary choice of a critical length and has proved to work well to predict crack initiation at Vnotches under symmetric [7] and complex loadings [12] in homogeneous materials. Williams' expansion (1) is used to derive the stress field and a matched asymptotic expansions procedure provides the energy release rate due to the onset of a short crack increment.

\section{Matched asymptotics}

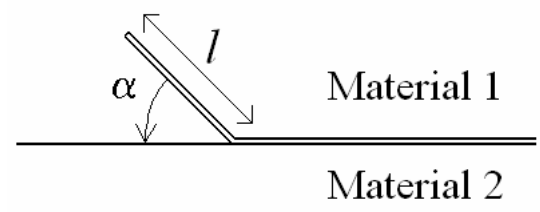

Figure 1. Crack kinking out of an interface.

The main difference with previous works is twofold, a complex singularity holds at the crack tip and the $T$-stress is taken into account in addition to singular modes. The crucial point lies in the comparison between two states: one relies on the initial structure, prior to crack initiation; the other refers to the same structure after onset of a short crack increment (length $\ell$ ) making an angle $\alpha$ with the primary crack (figure 1). The corresponding displacement fields are denoted respectively $\underline{U}^{0}(r, \theta)$ and $\underline{U}^{\ell}(r, \theta)$. The asymptotic procedure is carried out with respect to the small crack increment length and matching conditions with (1) lead to

$$
\left\{\begin{aligned}
\underline{U}^{0}(r, \theta)=\underline{U}^{0}(\rho \ell, \theta)= & \underline{R}+K \ell^{1 / 2+\mathrm{i} \varepsilon} \rho^{1 / 2+\mathrm{i} \varepsilon} \underline{u}(\theta)+\bar{K} \ell^{1 / 2-\mathrm{i} \varepsilon} \rho^{1 / 2-\mathrm{i} \varepsilon} \underline{u}(\theta)+T \ell \rho t(\theta)+\ldots \\
\underline{U}^{\ell}(r, \theta)=\underline{U}^{\ell}(\rho \ell, \theta)= & \underline{R}+K \ell^{1 / 2+\mathrm{i} \varepsilon}\left[\rho^{1 / 2+\mathrm{i} \varepsilon} \underline{u}(\theta)+\underline{V}(\rho, \theta)\right]+\bar{K} \ell^{1 / 2-\mathrm{i} \varepsilon}\left[\rho^{1 / 2-\mathrm{i} \varepsilon} \bar{u}(\theta)+\underline{V}(\rho, \theta)\right] \\
& +T \ell[\rho \underline{t}(\theta)+\underline{W}(\rho, \theta)]+\ldots
\end{aligned}\right.
$$

where $\rho=r / \ell$ holds for the dimensionless radial coordinate, and where the complex function $\underline{V}$ and the real one $\underline{W}$ are solutions to well-posed problems and vanish at infinity. They depend neither on the global geometry of the structure nor on the loading, and can be computed once and for all as functions of the crack initiation angle $\alpha$.

\section{The fracture criterion}

Using the previously described matched asymptotic analysis (section 2), it has been shown [7] that the initiation of a crack at a V-notch in a homogeneous material can be accurately predicted using two conditions involving both stress and energy. Furthermore, the proposed criterion coincides with Griffith's one for a pure crack. As a consequence of exponents greater than $1 / 2$ in the Williams' expansion in the general case, this initiation process is shown to be unstable; the crack jumps a short length. The presence of the $T$-stress term leads to a similar reasoning which is carried out herein.

Energy condition. The first condition results from an energy balance between two states of the structure prior and after the onset of a short crack increment. It states that the incremental energy release rate $G=-\delta W_{p} / \delta S$ has to exceed the toughness $G_{c}$ of the material, $\delta W_{p}$ being the elastic potential energy change and $\delta S$ the newly created crack surface. In plane elasticity, using equations (3) and (4) and asymptotic expansions with respect to the crack increment length $\ell(\delta S=\ell \times d$, where $d$ holds for the specimen thickness), the energy condition leads to

$$
G=K \bar{K} X(\alpha, m(\ell), M(\ell))+\ldots \geq G_{c}
$$


where $X$ is a coefficient depending on the crack initiation angle $\alpha$. It derives directly from the functions $\underline{V}$ and $\underline{W}$ and can be computed using the path-independent integral $H$ ([4], [9]).

Stress condition. The second condition is based on the maximum tension that a material can sustain before failure. It states that fracture can occur only if the opening stress along the expected crack path (defined above by the angle $\alpha$ and the length $\ell$ ) exceeds the material strength $\sigma_{c}$. Due to the oscillations, the forthcoming reasoning can be carried out using only an analogy with the approach used in the real case [8]. The stress condition $\sigma_{\theta \theta} \geq \sigma_{c}$ leads to the relation

$$
K \bar{K} Y(m(\ell), M(\ell))=\ell \sigma_{c}^{2} \quad \text { with } Y(m(\ell), M(\ell))=\left|s_{\theta \theta}(\theta)+m(\ell) s_{\theta \theta}(\theta)+M(\ell) \tau_{\theta \theta}(\theta)+. . .\right|^{2}
$$

where the index $\theta \theta$ stands for the hoop component of the tensors $\underline{\underline{s}}$ and $\underline{\underline{\tau}}$.

Mix criterion. The compatibility between (5) and (6) gives an equation for the crack initiation length $\ell_{c}$ as a function of $\alpha$

$$
\ell_{c} \frac{X\left(\alpha, m\left(\ell_{c}\right), M\left(\ell_{c}\right)\right)}{Y\left(m\left(\ell_{c}\right), M\left(\ell_{c}\right)\right)}=\frac{G_{c}}{\sigma_{c}^{2}}
$$

Finally eq. (5) with $\ell=\ell_{c}$ gives a condition on $|K|$ for crack initiation in the direction $\alpha$ :

$$
|K| \geq K_{\alpha}=\sqrt{\frac{G_{c}}{X\left(\alpha, m\left(\ell_{c}\right), M\left(\ell_{c}\right)\right)}}, \quad K_{f}=\operatorname{Min}_{\alpha} K_{\alpha}
$$

The critical value $K_{\alpha}$ depends on $\alpha$ and the actual kink angle $\alpha_{f}$ maximizes the denominator, i.e. minimizes $K_{\alpha}$ giving $K_{f}$ (i.e. $|K|$ at failure).

\section{Discussion}

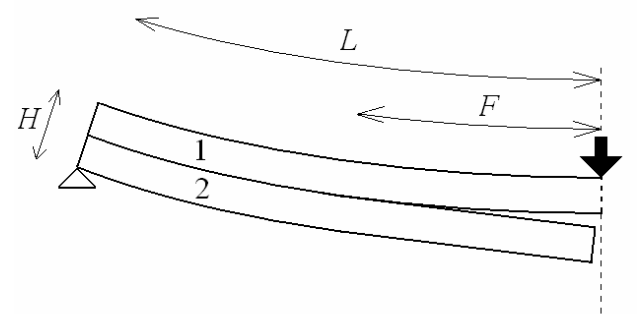

Figure 2. 3-point bending test, $F / L=0.25, H / L=0.2(1 / 2$ specimen $)$.

The kink of a crack in a homogeneous material has been widely studied in the literature, but it is difficult to find experiments to corroborate the predictions for a bimaterial. Many data are involved in the results: the elastic (Young's modulus $E$ and Poisson's ratio $v$ ) and fracture parameters (strength $\sigma_{c}$ and toughness $G_{c}$ ) of the two materials. Simulations are carried out on a bimaterial in flexion (figure 2), the stiffer material being alternatively in the upper $(R>1)$ and lower $(R<1)$ position, $R$ is the Young's modulus ratio, $v=0.3$ in both materials. Two cases are analysed: a weak and a strong contrast $E_{1}=300 \mathrm{GPa}, \sigma_{1 c}=400 \mathrm{MPa}$ and $G_{1 c}=0.05 \mathrm{MPa} . \mathrm{mm}\left(K_{I c}=4.06\right.$ $\left.\mathrm{MPa} . \mathrm{m}^{1 / 2}\right)$, and either $E_{2}=150 \mathrm{GPa}, \sigma_{1 c}=200 \mathrm{MPa}$ and $G_{1 c}=0.025 \mathrm{MPa} . \mathrm{mm} \quad\left(K_{I c}=2.03\right.$ MPa.m $\left.{ }^{1 / 2}\right)$ or $E_{2}=6 \mathrm{GPa}, \sigma_{1 c}=75 \mathrm{MPa}$ and $G_{1 c}=0.35 \mathrm{MPa} . \mathrm{mm}\left(K_{I c}=1.52 \mathrm{MPa} . \mathrm{m}^{1 / 2}\right)$. The stiffer material looks roughly like Alumina while the other looks either like a porous Alumina (weak contrast) or a polymer (strong contrast).

Results are in table 1. The presence of complex terms makes the interpretation quite entangled. A complete sensibility analysis is now in progress for a better understanding of the influence of the various parameters mentioned above. As already observed in the real case [8], $K_{f}$ defined in (8) 
decreases as $T$ increases (not illustrated herein). The particular case $R=1$ (no contrast) is given for comparison taking $K=K_{I}+\mathrm{i} K_{I I}$. It is worth noting that the sign of $\varepsilon$ is a convention since both + and - occur in eq. (1).

Table 1.

\begin{tabular}{|c|c|c|c|c|c|c|}
\hline$R$ & $\varepsilon$ & $\operatorname{Im}(K) / \operatorname{Re}(K)$ & $T / \operatorname{Re}(K)$ & $\alpha_{f}\left(^{\circ}\right)$ & $K_{f}\left(\mathrm{MPa} \cdot \mathrm{m}^{1 / 2}\right)$ & $\ell_{c}(\mu \mathrm{m})$ \\
\hline 1 & 0 & 0.93 & 1.29 & 60 & 2.27 & 17 \\
\hline 2 & 0.03 & 1.32 & 2.79 & 50 & 1.39 & 16 \\
\hline 0.5 & -0.03 & 0.89 & 1.33 & 60 & 0.88 & 18 \\
\hline 50 & 0.09 & 0.41 & 1.20 & 90 & 0.48 & 51 \\
\hline 0.02 & -0.09 & 1.58 & 0.38 & 70 & 0.81 & 68 \\
\hline
\end{tabular}

\section{Conclusion}

The $T$-stress together with the mix criterion defines an initiation length that allows getting rid of the oscillation terms in the prediction of the critical load and the deflection angle of a crack kinking out of an interface. For a complete analysis the competition between the deflection mechanism and the delamination growth must be analysed. It requires in addition the knowledge of the interface toughness, which determination is difficult, since it depends in particular on the modes mix.

\section{References}

[1] Cotterell B and Rice J R (1980) Slightly curved or kinked cracks. Int. J. Fract. 16(2):155-169.

[2] He M Y and Hutchinson J W (1989) Kinking of a crack out of an interface. J. Appl. Mech. 111:270-278.

[3] Kfouri A P (1986) Some evaluations of the elastic $T$-term using Eshelby's method. Int. J. Fract. 30:301-315.

[4] Labossiere P E W and Dunn M L (1998) Calculation of stress intensities at sharp notches in anisotropic media. Eng. Frac. Mech. 61(5-6):635-654.

[5] Larsson S G and Carlsson A J (1973) Influence of non-singular stress terms and specimen geometry on small-scale yielding at crack tips in elastic-plastic materials. J. Mech. Phys. Solids 21:263-277.

[6] Leevers P S and Radon J C (1982) Inherent stress biaxiality in various fracture specimen geometries. Int. J. Fract. 19:311-325.

[7] Leguillon D (2002) Strength or toughness? A criterion for crack onset at a notch. Eur. J. Mech. A/Solids 21:61-72.

[8] Leguillon D. and Murer S. (2008) Crack deflection in a biaxial stress state, Int. J. Fract. submitted.

[9] Leguillon D and Sanchez-Palencia E (1987) Computation of singular solutions in elliptic problems and elasticity. J. Wiley, New-York Masson, Paris, pp121-128.

[10] Rice J R (1988) Fracture mechanics concepts for interfacial cracks. J. Appl. Mech. 55:98-103.

[11] Selvarathinam A S and Goree J G (1998) T-stress based fracture model for cracks in isotropic materials. Eng. Frac. Mech. 60(5-6):543-561.

[12] Yosibash Z, Priel E and Leguillon D (2006) A failure criterion for brittle elastic materials under mixed mode loading. Int. J. Fract. 141(1):289-310. 